\title{
Performance study of the ATLAS Forward Proton Time-of-Flight Detector System
}

\author{
Karel Černý, on behalf of the ATLAS Collaboration ${ }^{* \dagger}$ \\ Regional Centre of Advanced Technologies and Materials, Joint Laboratory of Optics of Palacký \\ University and Institute of Physics AS CR, Faculty of Science, Palacký University, 17. listopadu \\ 12, 77146 Olomouc, Czech Republic \\ E-mail: karel.cerny@upol.cz,karel.cerny@cern.ch
}

\begin{abstract}
The performance of the ATLAS Forward Proton Time-of-Flight (ToF) detector is studied using the ATLAS LHC data collected in the 2017 running period of LHC Run2. A study of efficiency and time resolution of the ToF is performed. Good time resolutions of individual ToF detector channels, ranging between $20 \mathrm{ps}$ to $50 \mathrm{ps}$ are found, although the efficiency observed is well below $10 \%$ in major parts of the analysed data. The events from ATLAS physics runs at moderate pile-up taken at the end of 2017 are selected with signals in the two opposite ToF detector stations located at both sides of the ATLAS interaction region. The overall time resolution based on resolutions of the individual channels in these data is found to be $20 \pm 4 \mathrm{ps}$ and $26 \pm 5 \mathrm{ps}$ for the two ToF detectors. This represents a superb time resolution for a detector operating a few millimetres from the LHC beams. The difference of the primary vertex z-position measured by ATLAS and the value obtained by the ToF detectors is studied. The distribution of the difference constitutes of a background component from combinatorics since the level of pile-up is not negligible and a significantly narrower signal component from events where protons from interactions taking place in the primary vertex are detected in ToF. The fits are performed to the distributions yielding resolution of about $5 \mathrm{~mm} \pm 1 \mathrm{~mm}$ which is in agreement with the expectation based on singlechannel resolutions.
\end{abstract}

The 28th International Workshop on Vertex Detectors - Vertex2019

13-18 October, 2019

Lopud, Croatia

\footnotetext{
* Speaker.

$\dagger$ The work was supported by the Operational Programme Research, Development and Education - European Regional Development Fund, project no. CZ.02.1.01/0.0/0.0/16 19/0000754, of the Ministry of Education, Youth and Sports of the Czech Republic.
} 


\section{Introduction}

Observation of protons at high rapidities carrying large fraction of the initial state proton momentum serves as a signature of reactions $p p \rightarrow p X p$ commonly referred to as diffractive or photon induced processes. The LHC [1] optics separates the scattered protons from the beam to such extent that the deviations can be measured. For this purpose there are four ATLAS Forward Proton (AFP) detectors installed in pairs at $\sim 200 \mathrm{~m}$ on both sides of the ATLAS interaction point [2]. Each of the AFP detectors is equipped with Silicon tracker (SiT, [3]) which measures the positions $(x, y)$ and also angles $\left(x^{\prime}, y^{\prime}\right)$ of the scattered proton trajectory with respect to the nominal beam. These measured parameters are correlated with leading proton kinematics in the interaction point, see Figure 1. In case of pile-up (measured in terms of mean number of interactions per bunch crossing, $\langle\mu\rangle$ ) the detection of leading protons becomes too complicated due to combinatorial background. The information about the primary vertex position of the $p p \rightarrow p X p$ event can be, however, extracted by comparing the arrival time of the scattered protons. For this purpose the two outermost AFP stations called FAR-C and FAR-A for clockwise and anticlockwise directions, respectively, are equipped with ToF detectors.

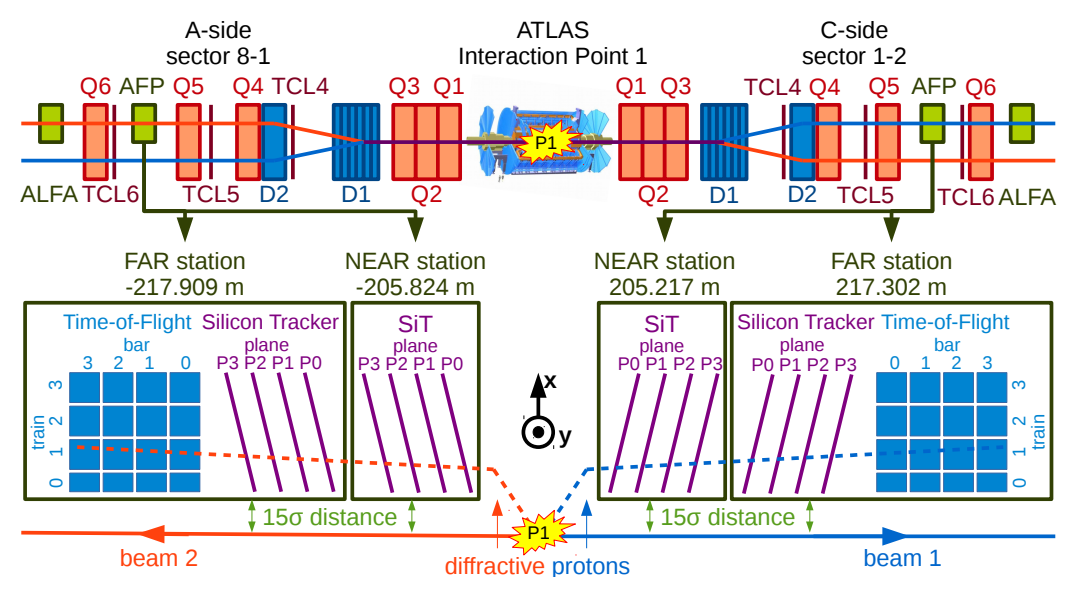

Figure 1: The general of AFP layout in ATLAS, see [2] and [1] for details.

\section{Description of the Time-of-Flight detector}

The ToF is an optical detector, collecting the Cherenkov photons produced in Quartz bars. Each bar consists of two arms, the radiator arm (exposed to the scattered protons) and the lightguide one both glued together forming the L-shaped Quartz bars (LQ-bars, [4]). The set of four LQ-bars (labelled as A,B,C,D) stacked along the beam axis is called a train. There are four trains placed on top of each other providing an extra spatial segmentation of the ToF detector. The resulting $4 \times 4$ matrix of the LQ-bar lightguides is attached to the micro-channel-plate photomultiplier (MCP-PMT, [5]) producing a pulse which is after amplification processed by the constant fraction discriminator (CFD) and high-performance time-to-digital converter (HPTDC). See Figures 2 and 3 to get a notion of the detector design and the ToF method. 

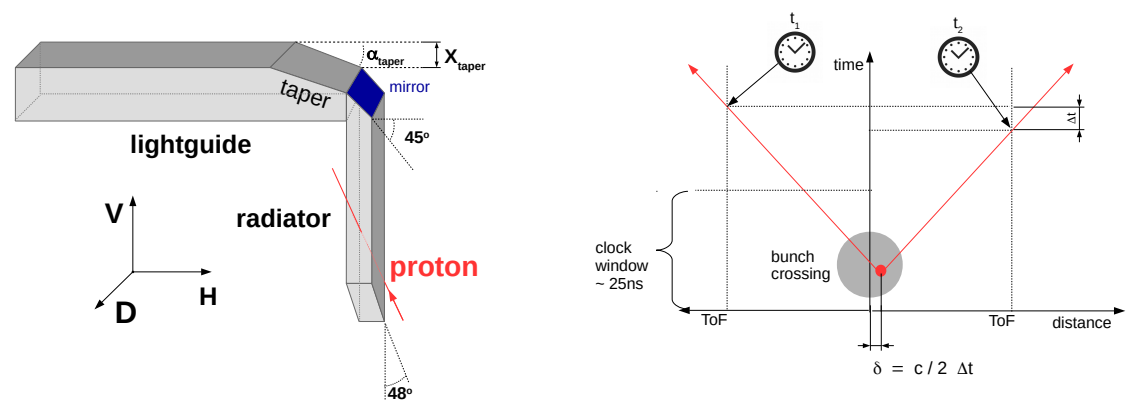

Figure 2: Left figure: the design of the LQ-bar. Right figure: the principle of measurement of the interaction vertex position using ToF.

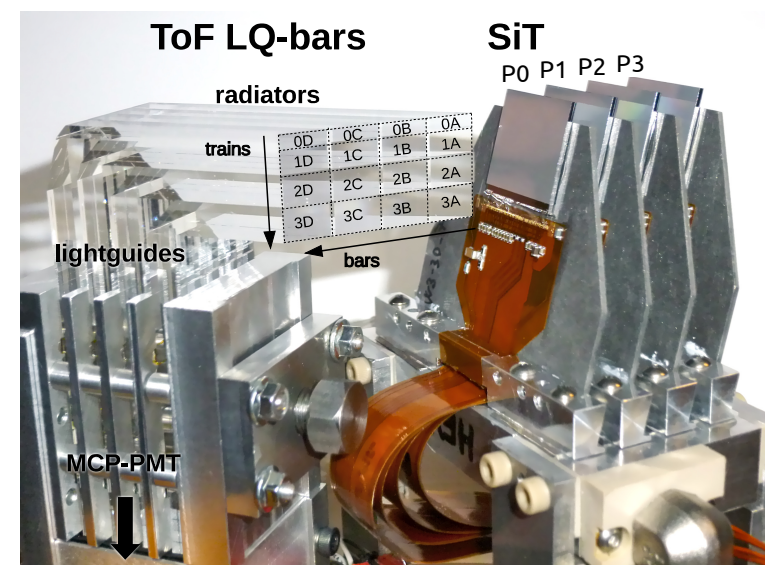

Figure 3: The assembled ToF LQ-bars and the SiT.

\section{Data}

The analysed data were recorded in 2017 by the ATLAS detector. The efficiency and singlechannel resolution are measured in the low- $\langle\mu\rangle$ runs 331202 and 336505 and also in run 336506 at a high- $\langle\mu\rangle$. Only the data from the AFP calibration stream, recorded with a SiT based trigger condition, were used. The same high voltage of $-2000 \mathrm{~V}$ was applied to the ToF PMTs in these runs.

For the vertex matching analysis a late 2017 run number 341419 was used taken in moderate pile-up conditions of $\langle\mu\rangle \sim 2$ with ToF PMTs high voltage of $-1950 \mathrm{~V}$ using a SiT based trigger. The AFP data were recorded together with the physics data from the central detector in the run 341419 .

\section{Measurement of efficiency}

Event samples where single track criteria are imposed on the SiT are used for measurement of 
the single channel efficiency defined as

$$
\varepsilon_{i k}=\frac{N\left(\text { channel }_{i k} \cap \operatorname{track}_{k}\right)}{N\left(\operatorname{track}_{k}\right)}
$$

where the first index $i$ labels the channel corresponding to the bar position in the train $k$ and the $\operatorname{track}_{k}$ refers to events with reconstructed SiT tracks physically pointing to the train $k$. The train efficiency is obtained using a logical OR over the trains' channels.

\section{Measurement of time resolution in single channels}

The time measured by a single channel reads, $t_{i}=t_{i, \text { proton }}+t_{i \text {,delay }}+t_{i, \text { smear }}-t_{\text {clock }}$, where the $t_{\text {proton }}$ is the true proton arrival time, $t_{\text {clock }}$ is the reference clock signal, $t_{i, \text { delay }}$ is a constant time offset due to signal path length in the electronics specific to each channel and $t_{i, \text { smear }}$ is the contribution smeared by all stochastic effects that play a role. It is the variance of $t_{i, \text { smear }}$ which defines the single-channel resolution. The resolutions are measured by using other bars of the same train as a reference. The time differences $\Delta t_{i j}=t_{i}-t_{j}$ are measured on the event-by-event basis in events where the signal is present in a single train only. The widths, $\sigma_{i j}$, of the $\Delta t_{i j}$ distributions given as $\sigma_{i j}^{2}=\operatorname{Var}\left(\Delta t_{i j}\right)$ are parameterised as $\sigma_{i j}^{2}=\sigma_{i}^{2}+\sigma_{j}^{2}-2 \rho_{i j} \sigma_{i} \sigma_{j}$, where $\sigma_{i}$ represent the single-channel resolutions and the $\rho_{i j}$ is a correlation factor between the two 'smear' times. The resulting single channel resolutions minimise the $\chi^{2}$ value defined as

$$
\chi^{2}\left(\left\{\sigma_{i}\right\}_{i=\mathrm{A}, \mathrm{B}, \mathrm{C}, \mathrm{D}}\right) \equiv \sum_{i \neq j} \frac{\left(\sigma_{i j}-\sqrt{\sigma_{i}^{2}+\sigma_{j}^{2}-2 \rho_{i j} \sigma_{i} \sigma_{j}}\right)^{2}}{\left(\delta_{\mathrm{fit}} \sigma_{i j}\right)^{2}},
$$

where $\delta_{\mathrm{fit}} \sigma_{i j}$ is the statistical uncertainty of the $\sigma_{i j}$ value obtained from fits to $\Delta t_{i j}$ distributions. The $\rho_{i j}$ are assumed to be zero varied by \pm 0.2 for evaluation of systematics where the variations lead to results statistically consistent with the nominal ones.

\section{Analysis of matching primary and Time-of-Flight vertex}

The $p p \rightarrow p X p$ interaction vertex position, $z_{\mathrm{ToF}}$, is reconstructed from proton arrival times measured by the ToF detector. The resolution of the $z_{\text {ToF }}$ is evaluated by measuring distribution of $z_{\text {ATLAS }}-z_{\text {ToF }}$, where $z_{\text {ATLAS }}$ is the primary vertex $z$-position measured by the central ATLAS detector. In case of pile-up the distribution of $z_{\text {ATLAS }}-z_{\text {TOF }}$ also contains the background contribution from random coincidences of protons measured in ToF not originating in a single interaction. By utilising the measured times from non-related events (event mixing) the shape of the background component is well approximated.

\section{Results}

The measured single-channel and train efficiencies are presented in Figure 4 for run 331020 based on a statistics of about $2 \mathrm{M}(3 \mathrm{M})$ tracks reconstructed in the FAR-A(C) station. The horizontal magenta boxes indicate in which train the SiT track was observed. The train efficiencies 
are shown by values in the last column (separated by vertical magenta line). The train efficiencies are obtained by requiring at least one channel hit in the given train. A general feature of the applied selection, presumably due to production of secondary particles, is that with a non-negligible probability signals can be generated also in bars of trains neighbouring the SiT-track train. The efficiencies measured in the SiT-track containing trains vary between $6-9 \%$ and $3.5-5 \%$ in stations FAR-A and FAR-C, respectively, in the run 331020. The difference between the efficiencies measured on both sides can be explained by unequal performance of the PMTs in the FAR-A and FAR-C stations.

In Figure 5 the efficiencies in trains with track are shown as a function of time in the runs 331020,336505 and 336506. An efficiency drop due to a continuous MCP-PMT degradation is observed between the run 331020 and the two runs 336505 and 336506 as the latter two ones were recorded after another two months of data taking after 331020. The statistics of track events amounts to $1 \mathrm{M}(7 \mathrm{M})$ in the run 336505 and to $60 \mathrm{k}(40 \mathrm{k})$ in the run 336506 for the FAR-A(C) side. Within the large uncertainties the efficiencies in the runs 336505 and 336506 do not depend on the value of $\langle\mu\rangle$ indicated with the magenta line.

The single-channel time resolutions are presented in Figure 6 for the runs 331020 and 336505. The number of single-train-topology events used for the measurement is $70 \mathrm{k}(110 \mathrm{k})$ in the run 331020 and $20 \mathrm{k}(100 \mathrm{k})$ in the run 336505 for the FAR-A(C) side. Due to the LQ-bar design and also verified by simulations it is known that photon leakage occurs between the LQ-bars downstream the proton motion, leading to a gradual photon enrichment of the latter bars. The worst resolutions of $40-50$ ps are observed in the A channels of the trains which have lowest photon yield. The following bars gradually profit from the photon enrichment yielding resolutions of $30-20 \mathrm{ps}$ of the $\mathrm{B}$ and $\mathrm{C}$ channels. Despite the photon enrichment from the previous bars, the resolution measured in the $\mathrm{D}$ channel is systematically worse in all trains. The effect can be explained by lower signal amplitude of the last train channels as the charge sharing between channels is weaker for the $\mathrm{D}$ channel. The same effect worsens the resolutions in the A channels. The total uncertainty equals to $6 \mathrm{ps}$ at most, covering both the statistical and systematic errors.

The vertex matching analysis is performed by fits to the $z_{\text {ATLAS }}-z_{\text {ToF }}$ distributions using a double-Gaussian p.d.f. accounting for the signal and background components. In Figure 7 the distributions of $z_{\text {ATLAS }}-z_{\text {ToF }}$ measured in events with ToF signals on both sides of the interaction region in run 341419 , where $z_{\text {ATLAS }}$ is the primary vertex $z$-position reconstructed by ATLAS. The distributions shown in figures a)-c) correspond to ATLAS data containing a reconstructed primary vertex together with coincidence of signals in both ToF detectors in three cut scenarios with respect to number of vertices reconstructed by ATLAS, no $N_{\mathrm{vtx}}$ cut, $N_{\mathrm{vtx}} \leq 5$ and $N_{\mathrm{vtx}} \leq 3$, respectively. A double Gaussian function representing the signal and background components is fitted to unbinned data samples using the extended likelihood fit as implemented in RooFit in all $N_{\mathrm{vtx}}$ cut scenarios. The mean of the signal component as well as the mean and width of the background component are always estimated from a Gaussian fit to the mixed event data in each $N_{\mathrm{vtx}}$ cut scenario separately, denoted as $\mu_{\mathrm{sig}}^{\mathrm{FIX}}, \mu_{\mathrm{bgd}}^{\mathrm{FIX}}$ and $\sigma_{\text {bgd }}^{\mathrm{FI}}$. The mixed event data $z_{\text {ATLAS }}-z_{\text {ToF }}$ distributions are obtained by random mixing of times measured by ToF in either station and the $z_{\text {ATLAS }}$ values which do not originate in the same collision event. The expected resolution of the ToF detector, quoted as $\sigma_{\text {expected }}^{\text {ToF }}=4.9 \pm 0.7 \mathrm{ps}$, is obtained from the known single-channel resolutions convoluted with the actual channel-hit-patterns observed in the data in the no $N_{\mathrm{vtx}}$ cut scenario [6]. The signal width 


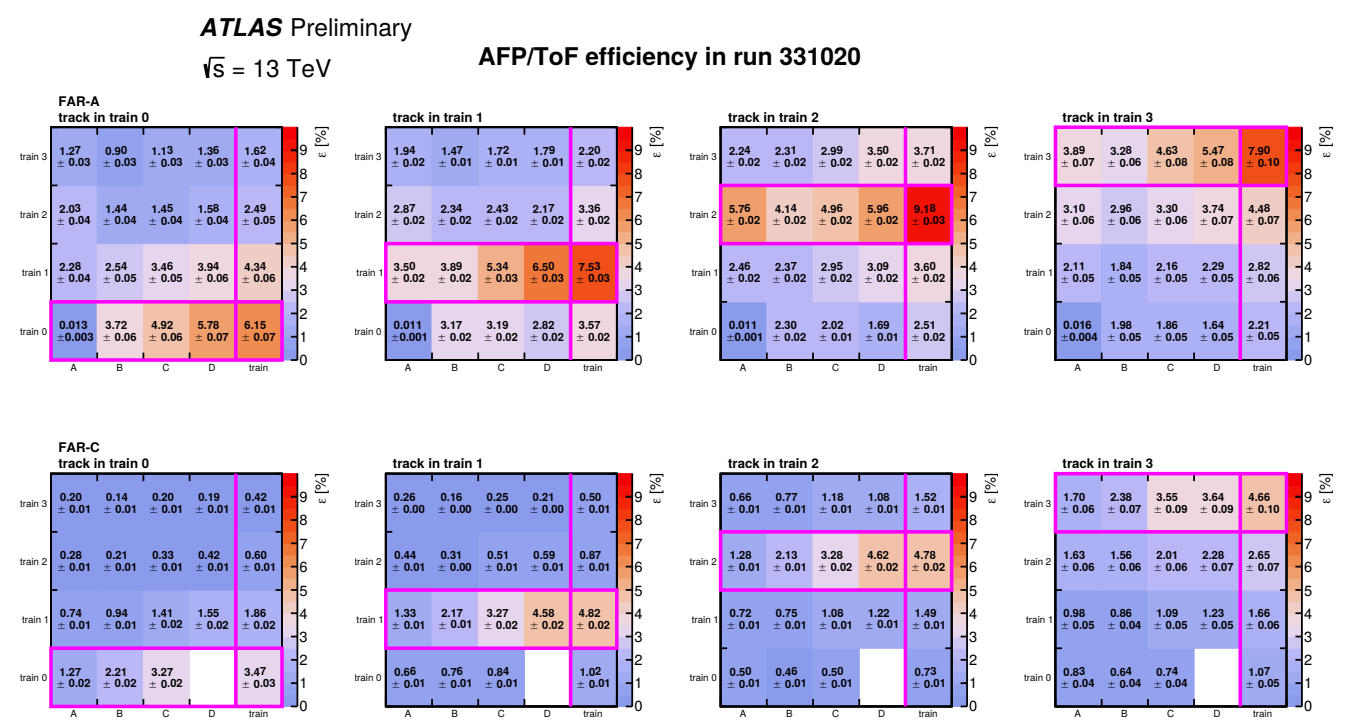

Figure 4: The ToF single-channel and train efficiencies in run 331020 [6].
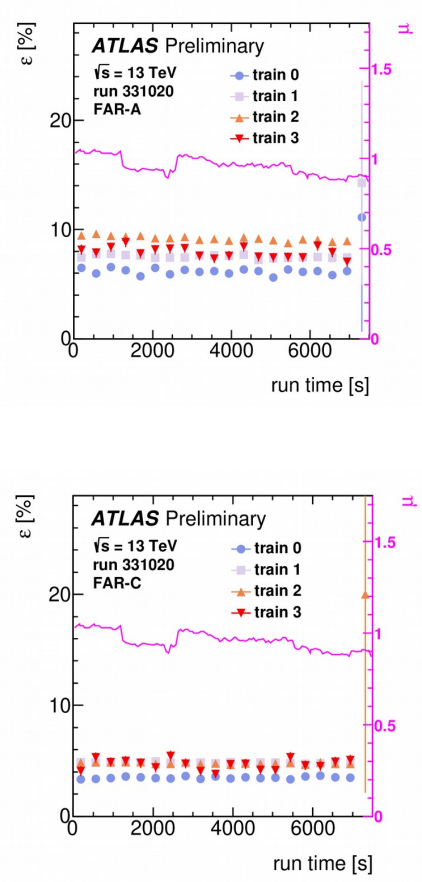
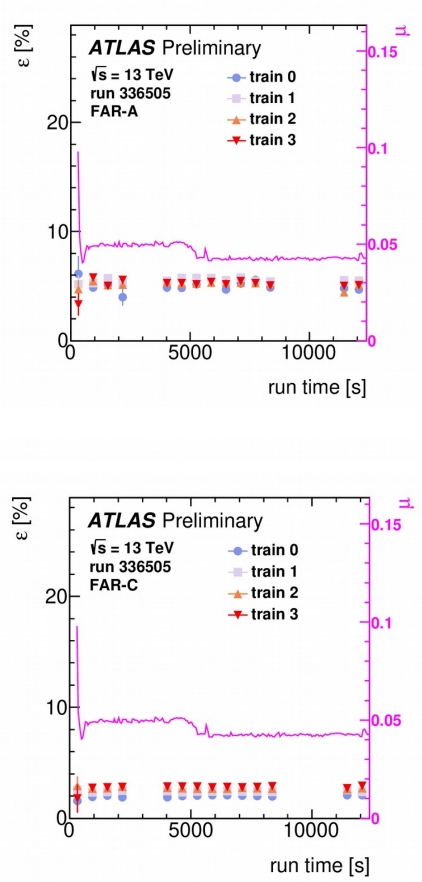
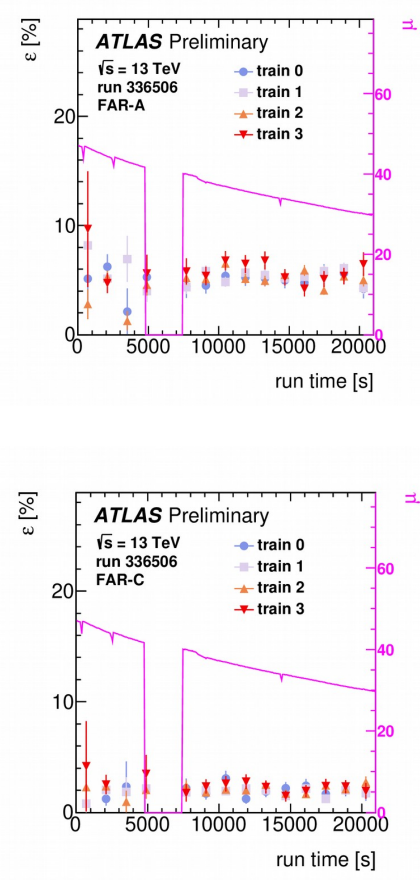

Figure 5: The ToF train efficiencies in the low- $\langle\mu\rangle$ runs 331020,336505 and the high- $\langle\mu\rangle$ run 336506 as a function of time in run [6]. 


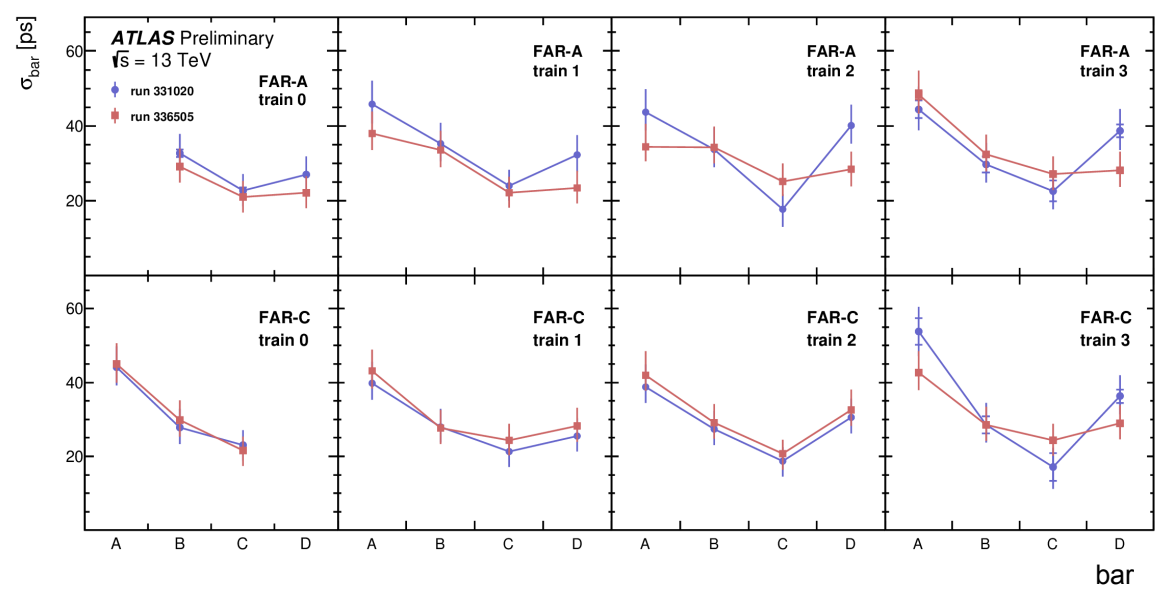

Figure 6: The single-channel resolutions in runs 331020 and 336505 [6].

obtained in three cut scenarios with respect to the $N_{\mathrm{vtx}}$ cut are within uncertainties in agreement with the value calculated from the single-channel resolutions, i.e. $\sigma$ (no $N_{\mathrm{vtx}}$ cut $)=5.2 \pm 0.9 \mathrm{ps}$, $\sigma\left(N_{\mathrm{vtx}} \leq 5 \mathrm{cut}\right)=5.0 \pm 0.8 \mathrm{ps}, \sigma\left(N_{\mathrm{vtx}} \leq 3 \mathrm{cut}\right)=6.0 \pm 1.0 \mathrm{ps}$. The differences in the extracted signal widths suggest the fits are sensitive to the statistics of the data. Nevertheless, the signal to background fraction $\left(n_{s} / n_{b}\right)$ is found to grow from $0.03 \pm 0.01$ to about $0.06 \pm 0.01$ if the loose (no $N_{\mathrm{vvx}}$ cut) or tightest $\left(N_{\mathrm{vtx}} \leq 5\right)$ cuts are applied. This is in accordance with expectation as the fraction of background naturally decreases with the number of additional interactions in a single bunch crossing.
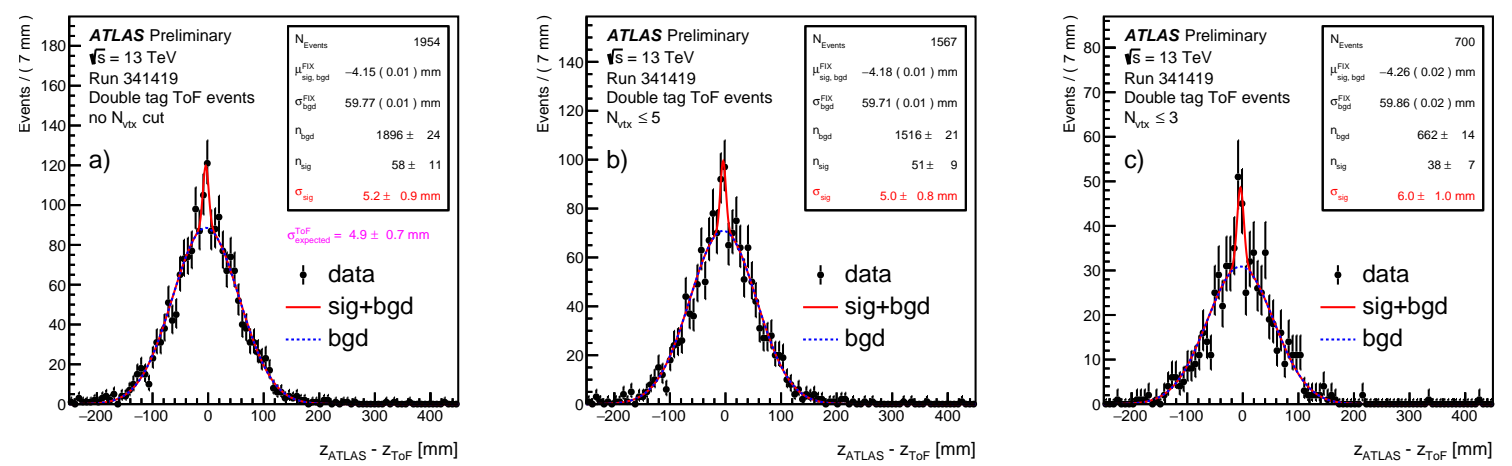

Figure 7: The distributions of $z_{\mathrm{ATLAS}}-z_{\mathrm{ToF}}$ measured in events with ToF signals on both sides of the interaction region in run 341419. Fits of signal and background components to the data obtained in three $N_{\mathrm{vtx}}$ cut scenarios are performed shown with the solid red and blue dashed line.

\section{Conclusions}

The performances of the ATLAS Forward Proton ToF detector have been studied using dedicated runs collected during the 2017 LHC data-taking. 
While the measured single-channel time resolutions are promising, ranging between $20 \mathrm{ps}$ and $50 \mathrm{ps}$, the ToF performance is burdened mainly by low efficiency of $1-7 \%$ in single channels and $5-10 \%$ in trains caused by a continuous degradation of the MCP-PMTs with collected charge. Improvements of all parts of the timing detectors are under development including the use of longlife MCP-PMTs and a new (glue-less) production technique of the LQ-bars.

Observation of a significantly narrower peak in the $z_{\mathrm{ATLAS}}-z_{\mathrm{TOF}}$ distribution provides a hint of presence of the signal $p p \rightarrow p X p$ events. The width of the extracted signal peak is between 5 and $6 \mathrm{~mm}$ which is broadly consistent with the expected resolution $4.9 \pm 0.7 \mathrm{~mm}$.

\section{References}

[1] L. Evans and P. Bryant, "LHC Machine," JINST 3 (2008), S08001.

[2] L. Adamczyk et al. "Technical Design Report for the ATLAS Forward Proton Detector," CERN-LHCC-2015-009.

[3] J. Lange et al.,"3D silicon pixel detectors for the High-Luminosity LHC,” JINST 11 (2016) no.11, C11024.

[4] L. Nozka et al., "Design of Cherenkov bars for the optical part of the time-of-flight detector in Geant4", Optics Express Vol. 22, Issue 23, pp. 28984-28996 (2014).

[5] PHOTONIS USA Pennsylvania Inc., mini PLANACON XPM85112 datasheet.

[6] https://twiki.cern.ch/twiki/bin/view/AtlasPublic/ForwardDetPublicResults 